\title{
On the Intrinsic Thermal Stability in HVDC Cables
}

\author{
Ch. Chakradhar Reddy and T. S. Ramu \\ Department of Electrical Engineering \\ Indian Institute of Science \\ Bangalore, PIN: 560 012, India.
}

\begin{abstract}
The paper deals with some important aspects of steady state thermal stability in the insulation of HVDC cables. The resistively graded electric field distribution and the temperature distribution, under steady state, in dc cables, are interrelated. The dc conductivity is known to be a function of stress as well as of temperature. Reasonably convincing theories of electric stress and temperature distribution are now available in the literature. Until recently, the thermal runaway was believed, primarily, to be a consequence of an inordinate run away in the sheath temperature due to dynamic interaction with the ambient. While this premise is partly true, there are other overriding factors controlling the stability. This paper presents a detailed investigation on this aspect and it is shown that the multi-factor dependent de conductivity alone causes the thermal instability, boundary conditions at sheath having a marginal role.

Index Terms - Insulation, thermal breakdown, HVDC cable.
\end{abstract}

\section{NOMENCLATURE}

$E(r) \quad$ the electric field at radial position $r$

$T(r)$ the temperature at radial position $r$

$\sigma(r) \quad$ the dc conductivity at radial position $r$

$I$ the insulation leakage current per unit length

$I_{L} \quad$ the conductor current

$r_{l} \quad$ the radius of the conductor

$r_{2}$ the radius of the outer surface of the insulation

$k_{i} \quad$ the thermal conductivity of the insulation

$R$ the conductor resistance per unit length of the cable

$R_{i} \quad$ the insulation resistance per unit length of the cable

$E_{m} \quad$ the maximum stress in the insulation

$T_{m} \quad$ the maximum temperature in the insulation

$T_{a} \quad$ the ambient temperature $\left(25^{\circ} \mathrm{C}\right)$

MITB the value of $T_{m}$ at the onset of thermal runaway

$\Delta \theta \quad$ the contribution of insulation leakage current loss to $T_{m}$

$\Delta \theta_{c} \quad$ the value of $\Delta \theta$ at the onset of thermal runaway

\section{INTRODUCTION}

THE intrinsic and interactive thermal instability in electrical insulation has been a topic of great interest for some time now. Eoll [1] treated this subject in some detail while dealing with dc cables.

Manuscript received on 12 February 2007, in final form 7 June 2007.
Efforts aimed at distinguishing the implied instability have lead to the current status of research in this area. Despite the earlier impetus, interest in this very important aspect seems to have waned.

Among the other earlier authors, Fallou [2], worked on the subject of interactive thermal instability in cable insulation while Whitehead [3] and O'Dwyer [4] were known for their work on the thick dielectric slab of plane parallel geometry. Referring to Figure 1 , it depicts the concept of thermal runaway (in sheath temperature and a consequent runaway in the bulk insulation), from which a formal definition emerges for interactive thermal instability as a condition in which the insulation does not attain equilibrium with respect to the ambient. This statement, however, presupposes a constant, non-zero, external thermal resistance.

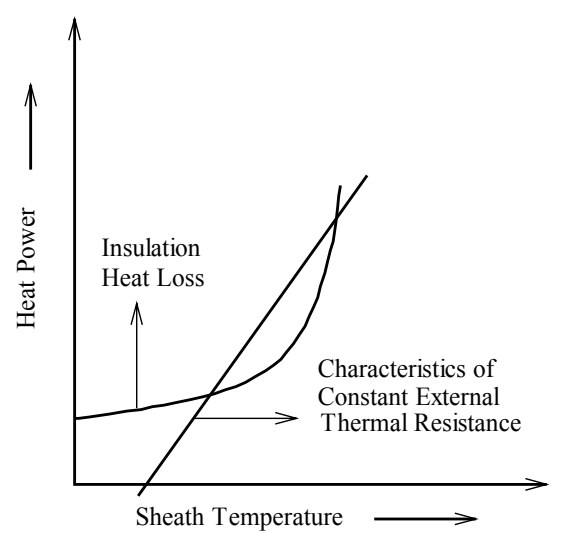

Figure 1. Depiction of interactive thermal instability.

The second kind of instability (intrinsic thermal instability) mentioned by Eoll in, for example, dc cable insulation is seen to 
have been ignored by other authors. Even in the absence of an external interaction, thermal instability may occur within the cable insulation [1]. In an earlier work of the authors of this paper, [5], a solution to the generic boundary value problem for thermal and electrical continuity was proposed. As applied to a dc cable, when the sheath temperature is maintained at a known temperature, say, by a constant temperature infinite heat source/sink, intrinsic thermal instability can be visualized.

The thermal maximum voltage for a thick slab of dielectric included between plane parallel electrodes has been investigated by Whitehead and O'Dwyer [3, 4] treating the electrode surface boundary temperature as a constant. The thermal maximum voltage, referred to in those works, is shown here to be different from the author's intrinsic thermal stability limits.

Referring to Figure 2, the steady state thermal maximum voltage, $V_{\max }$, assumes importance due to the reason that this is the maximum voltage below which the thermal runaway can not occur even in the remote future after an application of voltage [3, 4]. For a few percent higher voltages than this the thermal runaway may result in a rapid breakdown. The fact that the application of voltage equal to the thermal maximum voltage results in the runaway at theoretically infinite time makes it difficult for laboratory experimentation or estimation.

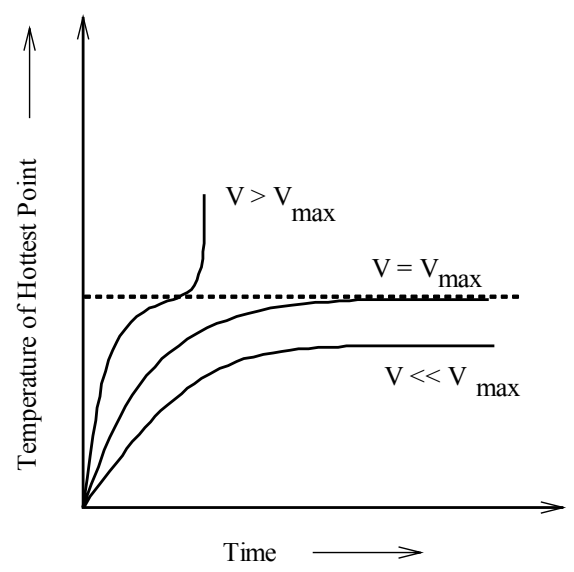

Figure 2. Depiction of runaway in the hottest point temperature.

The intrinsic instability as shown here, is, a consequence of the electrical conductivity being made a function of temperature and electric field. The coupled thermal and current continuity conditions, under stated boundary conditions, are seen to give double solutions to stress and temperature in the neighborhood of an inherent peak voltage referred to as the intrinsic thermal maximum voltage (ITMV). In so doing, the maximum insulation temperature at breakdown (MITB) and the effects of the thickness on the ITMV, which seem to have not been covered earlier, have been examined in detail. Several gaps existing in Eoll's formulations are now filled leading to a comprehensive understanding of the concept of intrinsic thermal stability.

\section{PHENOMENOLOGICAL MODEL}

For the sake of completeness and for a quick reference, the expressions for electric field, $E(r)$, the dc conductivity $\sigma(r)$ and the temperature, $T(r)$, in the insulation, as they appear in [5], are included in the following.

Several phenomenological formulations are available for dc conductivity, $\sigma(r)$, in the literature [6-10]. Authors have examined, at length, many empirical models for $\mathrm{dc}$ conductivity. The EPRI (Electric Power Research Institute) [8] model, given below is used in this work in view of its logical evolution and the representation given to both $E(r)$ and $T(r)$.

$$
\sigma(r)=A \cdot e^{a|E(r)|} \cdot e^{-b / T(r)}
$$

where

$$
\begin{aligned}
& A \text { is a material dependent constant } \\
& a \text { is known as stress coefficient } \\
& b \text { is known as temperature coefficient }
\end{aligned}
$$

Referring to Figure 3, for $r_{1}<r<r_{2}$, the temperature $T(r)$ and stress $E(r)$, are given below:

$$
T(r)=a_{1} I_{0}(2 \sqrt{\beta r})+a_{2} K_{0}(2 \sqrt{\beta r})+\frac{\alpha}{\beta}-\frac{\gamma}{\beta} \ln (r)
$$

And

$$
E(r)=\frac{-c_{2}}{2 c_{1}} \pm \sqrt{\frac{c_{2}^{2}}{4 c_{1}^{2}}-\frac{1}{c_{1}}\left(\ln \left(\frac{I}{2 \pi r A E_{0}}\right)+\frac{b}{T(r)}+\frac{3}{2}\right)}
$$

where

$a_{1}$ and $a_{2}$ are arbitrary constants to be determined by the thermal boundary conditions;

$\alpha, \beta, \gamma, E_{o}, c_{1}$ and $c_{2}$ are constants given in [5];

$I_{0}$ and $K_{0}$ are the modified Bessel functions of zero order.

Unless otherwise mentioned all computations in this work pertain to an example Cross Linked Poly Ethylene (XLPE) cable, for which, the geometrical, electrical and thermal parameters are given in Table 1.

Table 1. The parameters of example XLPE cable.

\begin{tabular}{|c|c|}
\hline Item & Value \\
\hline$r_{1}$ & $22.5 \mathrm{~mm}$ \\
\hline$r_{2}$ & $44.2 \mathrm{~mm}$ \\
\hline$R$ & $17.38 \times 10^{-6} \Omega / \mathrm{m}$ \\
\hline$k_{i}$ & $0.34 \mathrm{~W} / \mathrm{m} \mathrm{K}$ \\
\hline$A$ & $2.2896 \times 10^{-6}(\Omega . \mathrm{m})^{-1}$ \\
\hline$a$ & $0.142 \times 10^{-6}(\text { Volts } / \mathrm{m})^{-1}$ \\
\hline$b$ & $7600\left({ }^{0} \mathrm{~K}\right)$ \\
\hline
\end{tabular}




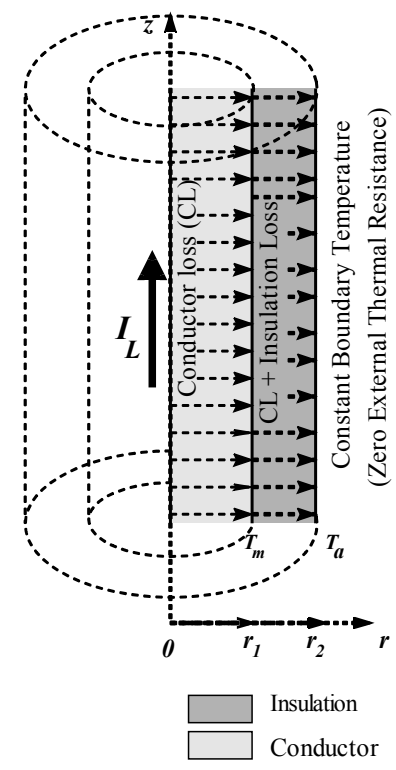

Figure 3. Boundary conditions for visualizing intrinsic thermal stability (Longitudinal cross sectional view of a cable depicting heat flow).

\section{THE INTRINSIC THERMAL INSTABILITY}

If the sheath temperature is dynamic (slowly changing with time) interactive thermal instability (Figure 1) is found to occur. If, on the other hand, the sheath temperature is held constant, an unstable state of the intrinsic type is reached. In both cases, the dc conductivity is a function of temperature and stress. All computations, in this work, are performed taking the magnitude of the sheath temperature as $25{ }^{\circ} \mathrm{C}$. These computations have actually been obtained using the authors' earlier work [5] (equations, (2) and (3)), when the external thermal resistance is equal to zero. This reflects an ideal case of infinite thermal conductivity of the medium surrounding the cable.

The corresponding boundary conditions can now be stated as follows:

Referring to Figure 3, the thermal boundary condition at $r=r_{1}$ is influenced by the conductor losses. Under steadystate, instantaneous heat generated will be crossing this boundary.

- At $r=r_{1}$, by Fourier Law [11] of heat conduction,

$$
\left.2 \pi r_{1} k_{i} \frac{d T(r)}{d r}\right|_{r=r_{1}^{+}}=-I_{L}^{2} R
$$

- At $r=r_{2}, T\left(r_{2}\right)=T_{a}=25^{\circ} \mathrm{C}$

In the following sections, detailed investigations on the causes of the intrinsic nature of thermal instability, its dependence on the insulation thickness and other related aspects, which seem to have not been covered in earlier works, are presented.

\subsection{The Causes of Instability}

The failure of Ohm's law ( $I \alpha V$ ) is borne out of the fact that $\sigma$ is a function of $E(r)$ in addition to being a function of $T(r)$. Interestingly, the complex phenomenon of intrinsic thermal stability can be explained with this simple fact below.

The dc conductivity at any radial position can be estimated using equations (1), (2) and (3), and hence, given $I$, the leakage current, the bulk insulation resistance, $R_{i}$, can be estimated.

$$
R_{i}=\int_{r_{1}}^{r_{2}} \frac{d r}{2 \pi r \sigma}=\frac{1}{2 \pi A} \int_{r_{1}}^{r_{2}} \frac{e^{-a E(r)+b / T(r)}}{r} d r
$$

The steady state voltage $\mathrm{V}$ across the insulation can be expressed in terms of leakage current and the non linear resistance $R_{i}$ as follows:

$$
V=R_{i} \times I
$$

where, $R_{i}$ is computed at constant sheath temperature. Referring to Figure 4, observe that $R_{i}$ is a monotonically decreasing function of the heat generated in the insulation due to leakage current (computed using equations (2), (3) and (5)). The product on the right-hand side of equation (6) can be shown to attain a maximum for a certain critical value of the leakage current (marked *, Figure 4) above which the decrement in $R_{i}$ is much more rapid than the increment in $I$ and the product tends towards lower values. The maximum voltage corresponding to this critical leakage current is the ITMV. An application of a voltage higher than this would result in thermal breakdown and for voltages less than this there are two solutions; a stable and an unstable state.

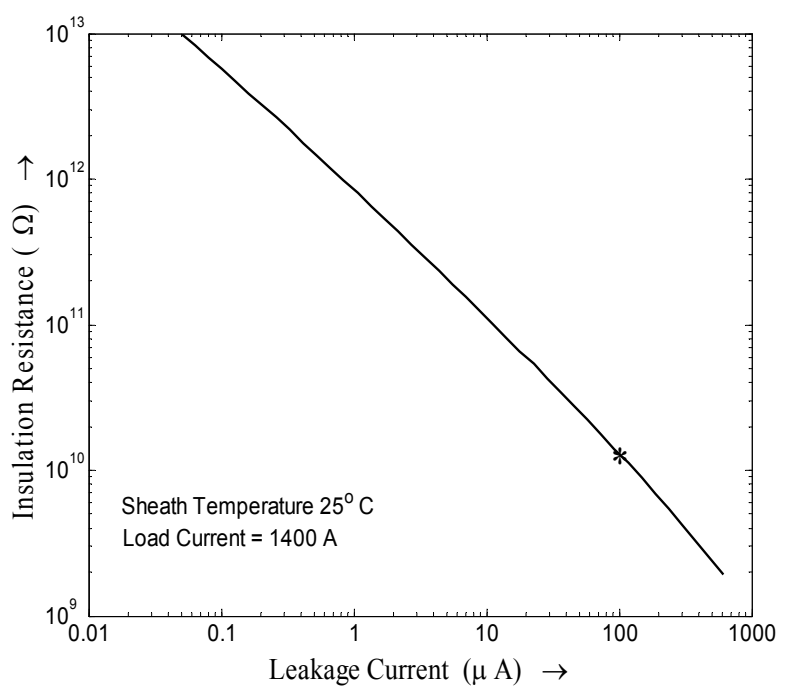

Figure 4. Insulation resistance vs leakage current. 
A substantiative explanation for the intrinsic instability emerges from the above discussion, thus:

> The intrinsic thermal stability limit arising out of this criticality is due to the synergic stress dependence of dc conductivity, Trace 1, Figure 5.

$>$ As a matter of fact, it can be shown, that, this aspect is invariant with respect to the stress dependent formula for the dc conductivity. It is interesting to observe that if $\sigma$ depends only on the temperature, the right-hand side of the equation (6) asymptotically approaches a finite maximum value (Trace 2, Figure $5)$.

$>$ It is a fact that the intrinsic thermal instability is independent of external thermal resistance. It is observed that a choice of higher sheath temperature results in a lower value of computed ITMV.

$>$ The ITMV assumes particular importance due to the fact that this happens to be the ultimate upper thermal limit of the voltage (Interactive thermal maximum voltage limit will always be less than this).

The interactive thermal voltage limits depend on external thermal resistance. It can be shown that, given a sheath temperature, intrinsic thermal maximum voltage is uniquely defined for a given cable. It is therefore interesting to note that ITMV serves as a useful index in the assessment of the relative thermal performance of $\mathrm{dc}$ cable insulation.

In view of the above observations, Table 2 attempts to bring out the differences between the two kinds of instability.

Table 2. Differences between Intrinsic and Interactive Stability.

\begin{tabular}{|c|l|l|}
\hline S.No & Intrinsic thermal instability & Interactive thermal instability \\
\hline 1 & $\begin{array}{l}\text { Does not depend on } \\
\text { surrounding medium. } \\
\text { Depends on internal thermal } \\
\text { resistance of the insulation. }\end{array}$ & $\begin{array}{l}\text { Depends on surface heat } \\
\text { transfer property of sheath as } \\
\text { well as on the thermal } \\
\text { resistance of the surroundings. }\end{array}$ \\
\hline 2 & $\begin{array}{l}\text { Occurs due to nonlinear dc } \\
\text { conductivity oftress } \\
\text { dependence) or failure of } \\
\text { Ohm's law in dielectrics }\end{array}$ & $\begin{array}{l}\text { Occurs primarily due to failure } \\
\text { of thermal equilibrium with } \\
\text { surroundings added with non- } \\
\text { linear de conductivity }\end{array}$ \\
\hline 3 & $\begin{array}{l}\text { If } \sigma \text { were a function only of } \\
\text { temperature, this kind of } \\
\text { instability may not occur }\end{array}$ & $\begin{array}{l}\text { It can be shown that, this kind } \\
\text { of instability occurs, even if } \sigma \\
\text { is a function only of } \\
\text { temperature. }\end{array}$ \\
\hline 5 & $\begin{array}{l}\text { Exhibits a stable and an } \\
\text { unstable state at two different } \\
\text { values of leakage current }\end{array}$ & $\begin{array}{l}\text { Exhibits a stable and an } \\
\text { unstable state at two different } \\
\text { values of sheath temperature }\end{array}$ \\
\hline $\begin{array}{l}\text { For higher voltages than } \\
\text { stable nor unstable solution }\end{array}$ & $\begin{array}{l}\text { For higher external thermal } \\
\text { resistance there exists neither a } \\
\text { stable nor an unstable solution }\end{array}$ \\
\hline
\end{tabular}

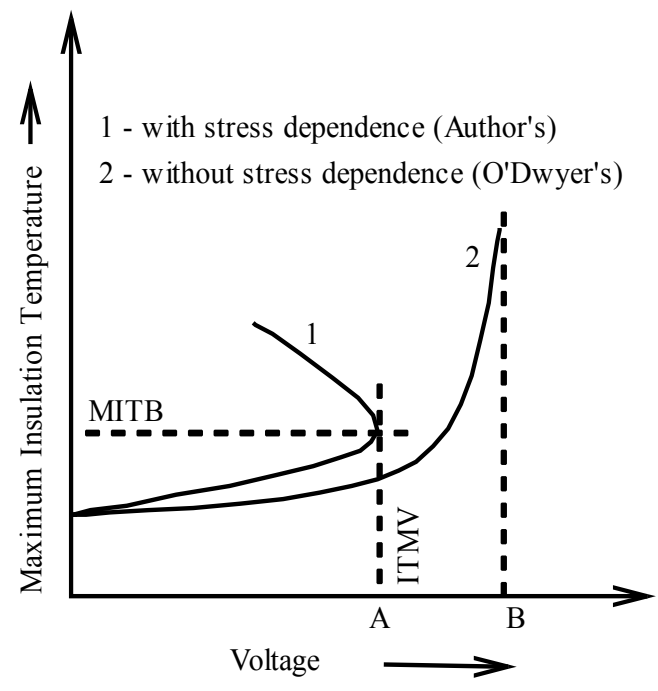

Figure 5. Depicting the effect of field dependent dc conductivity on maximum thermal voltage and intrinsic stability(At constant sheath temperature).

\section{DISCUSSION}

It is already mentioned, that, the thermal maximum voltage for a thick slab of dielectric included between plane parallel electrodes has been investigated by Whitehead and O'Dwyer [3, 4, 9] treating the electrode surface boundary temperature as a constant. This implies an ideal boundary, a boundary layer with infinite thermal conductivity. Interestingly, the thermal maximum voltage referred to in this case, is the same as the asymptotic value mentioned earlier in this work (ignoring the stress dependence of $\sigma$ ). As a matter of fact the intrinsic thermal maximum voltage, worked out by the Authors is considerably different from the asymptotic value. Figure 5 brings out this point succinctly. It may be observed there from, that, the ITMV indicated by the point A (on trace 1, by the Authors) may be seen to be much different from the corresponding point, B (trace 2, based on Whitehead or O'Dwyer suggestion). It is therefore felt appropriate to consider the ITMV (point A) in the design of dc cables.

\subsection{ITMV with Insulation Thickness}

The relationship between the thermal breakdown voltage and the thickness of insulation has been discussed at length by Whitehead [3]. It is generally believed that the thickness of insulation has only a marginal effect on the thermal maximum voltage. This statement is true for plane parallel geometry, provided that $\sigma$ is a function only of the temperature. However, due to the radial dependence of $\sigma$ on $E(r)$ and $T(r)$, under cylindrical geometry, the ITMV indeed depends on the thickness. This dependence is further enhanced by stress inversion occurring in the cable insulation. This aspect is demonstrated through the following computations.

The ITMV, $E_{m}, T_{m}$ and other quantities, computed using the authors' formulations keeping $r_{1}$ and sheath temperature constant (at $22.5 \mathrm{~mm}$ and $25{ }^{\circ} \mathrm{C}$ respectively) over a range of 
values of $r_{2}$ are plotted in Figures 6-10. Referring to these figures, the following critical observations can be made:

- Referring to Figure 6, the maximum temperature curves (computed using equation (2), putting $r=r_{l}$ ) are seen to have lifted up for increasing thickness. This is due to the fact that with thickness the thermal resistance of the insulation increases.

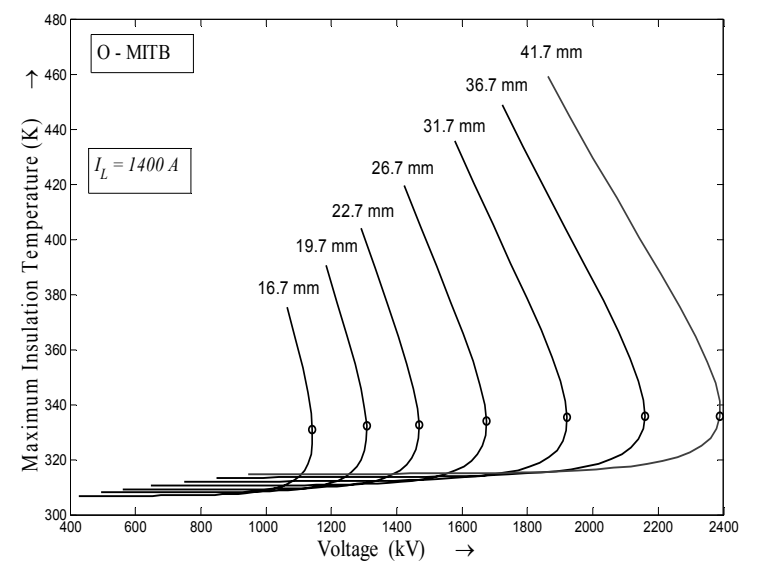

Figure 6. Maximum Insulation Temperature vs Voltage at different insulation thickness.

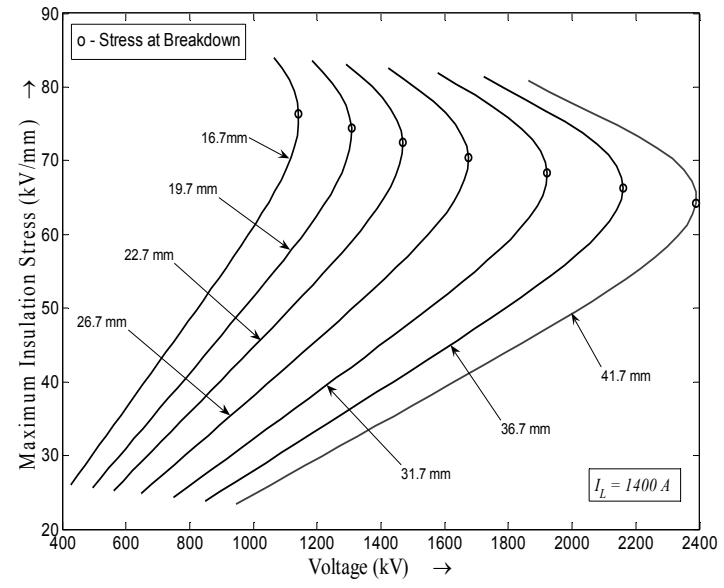

Figure 7 Maximum insulation stress vs voltage at different insulation thickness

- The maximum insulation stress (computed using equation (3), putting $r=r_{l}$ ) at thermal breakdown and the average thermal breakdown strength tends to decrease with the thickness in spite of the fact that the ITMV tends to increase (Figures 7, 8 and 10). In the limit, as thickness tends to infinity, both these appear, asymptotically, to approach finite values.

- Thermal breakdown is particularly important as the insulation thickness increases. It may be seen, from Figure 8, that the thermal breakdown strength falls considerably below the electronic breakdown strength $(\sim 100-130 \mathrm{kV} / \mathrm{mm}$ for XLPE).

- A very important fallout of this discussion is that, since the slope of the maximum insulation stress with voltage, decreases with thickness, the thicker cables are more robust in withstanding larger voltage fluctuations (Figure 7) or system over voltages.

- The critical leakage current (the leakage current corresponding to ITMV) decreases with the insulation thick ness (Figure 9).

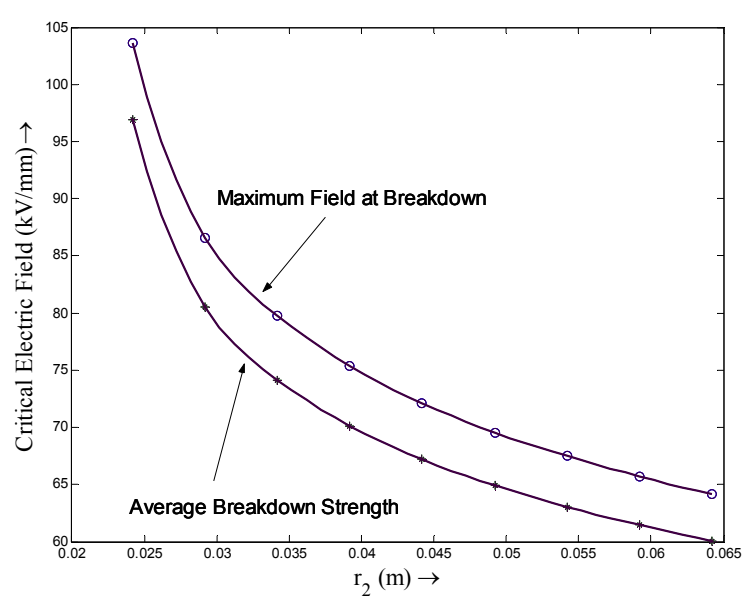

Figure 8. Maximum electric stress in the insulation at breakdown and average thermal breakdown strength vs thickness.

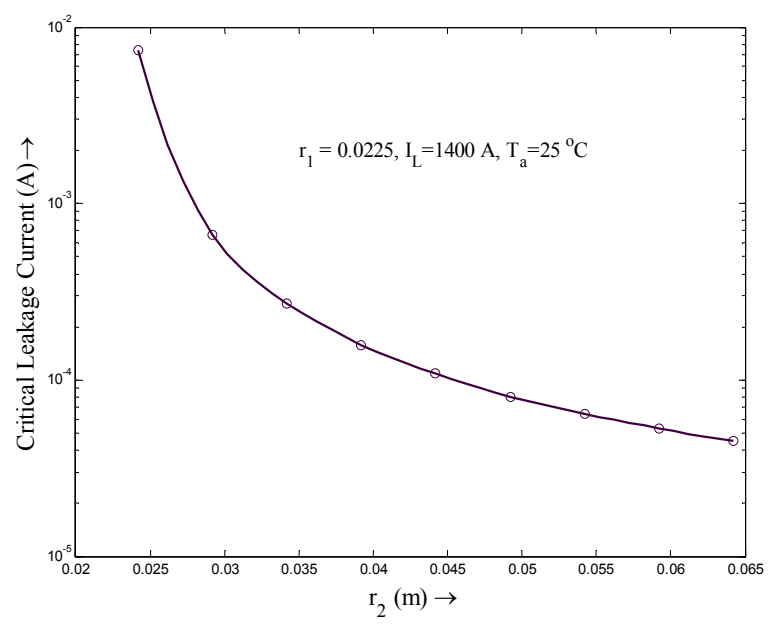

Figure 9. Leakage current at breakdown vs insulation thickness.

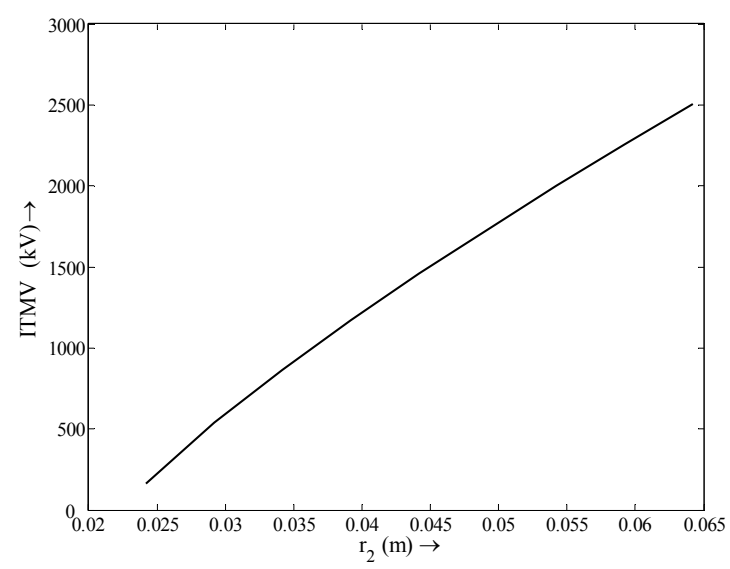

Figure 10. Thermal breakdown voltage vs insulation thickness. 


\subsection{The Critical Temperature (MITB)}

An interesting aspect of intrinsic thermal instability is the maximum insulation temperature at breakdown (MITB). It is observed that the maximum insulation temperature at breakdown is not very high, often, considerably lower than the decomposition and depends on the insulation thickness as well as the load current. According to Whitehead [3] the thermal breakdown could occur at temperatures as low as $10{ }^{\circ} \mathrm{C}$ above the boundary temperature. This temperature rise was solely due to heat produced by insulation leakage current (for example, a case of a cable with no load current). The author's present work throws more light on this aspect. The effect of external heat (conductor related heat) is shown to increase MITB.

To elucidate this aspect, computation of MITB is made for different load currents and thickness with constant sheath temperature, $25^{\circ} \mathrm{C}$. The solid curves in the Figures 11,12 and 13 correspond to the computation of MITB using equation (2) (putting $r=r_{l}$ ). In the following the role of insulation leakage current losses in the breakdown is investigated. It is to be noted that MITB constitutes of two components, viz. the contribution of conductor losses and the contribution of insulation leakage current losses.

Ignoring, for a moment, the insulation leakage current losses, the conductor boundary temperature can be shown to be:

$$
T_{c o n}=\frac{I_{L}^{2} R}{2 \pi k_{i}} \ln \left(\frac{r_{2}}{r_{1}}\right)+T_{a}
$$

where $T_{c o n}$ is purely the contribution of conductor losses to the $T_{m}$.

However, insulation losses do contribute marginally to conductor boundary temperature. If $\Delta \theta$ is the share of insulation leakage current losses in $T_{m}$, it can be expressed as:

$\Delta \theta=T_{m}-T_{c o n}$

Further, the breakdown can be construed as the condition satisfied by,

$$
\underset{T_{m} \rightarrow \text { LimB }}{\operatorname{Lim}}\left(T_{m}-T_{c o n}\right)=\Delta \theta_{c}
$$

Referring to Figures 11, 12 and 13, the dotted curves correspond to $T_{c o n}$ appearing in the equation (7). Significantly, the difference, $\Delta \theta_{c}$, between $T_{c o n}$ and MITB (the solid curves), can be seen to be sensibly constant to within $\sim \pm 2{ }^{\circ} \mathrm{C}$. This means that, irrespective of insulation thickness and load current, the breakdown occurs when the contribution of insulation leakage current losses, to the maximum insulation temperature, crosses a particular critical value $\Delta \theta_{c}$.

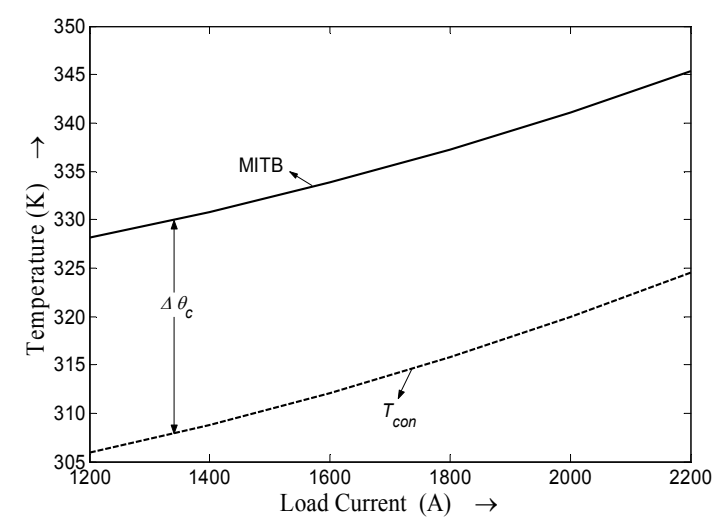

Figure 11. MITB and $T_{\text {con }}$ with load current $\left(r_{1}=22.5 \mathrm{~mm}, r_{2}=44.2 \mathrm{~mm}\right.$ and $T_{a}=25^{\circ} \mathrm{C}$, held constant).

As for the energy absorption in the insulation, when the incremental gain in internal energy (due to leakage i.e., mass * specific heat $* \Delta \theta$ ) exceeds a critical level at some point (hottest point), a breakdown occurs no matter what the thickness and boundary conditions are.

A possible upper limit can therefore be specified on net power (arising out of the electro-thermal effects in insulation) from a unit volume and represents a notional critical limit.

The results of this research work reveal an important fact that, $\Delta \theta$, being dependent almost entirely on the $\sigma$ and $k$, appears to be a plausible parameter for prediction of breakdown. This is possible if the information regarding conductor loading, conductor temperature $\left(T_{m}\right)$, and the sheath temperature are made available. The closer the value of $\Delta \theta$ to $\Delta \theta_{c}$ the higher is the probability of failure in the nearest future. Since the processes leading to breakdown, are, initially, very slow, an accurate prediction of $\Delta \theta$ can always prevent thermal failure.

It may also be pointed out that a class of insulating materials for dc cable application may be assessed based on their thermal stability limit, in other words, on the value of $\Delta \theta_{c}$. For example, for an XLPE cable insulation, with higher activation energy than the cable considered earlier in this work (all other parameters being same), the value of $\Delta \theta_{c}$ is smaller as shown in the Table 3. It is important to note that the MITB is dependent on the value of the sheath temperature, in spite of the fact that $\Delta \theta_{c}$ is independent of it. For instance, for cable 1 , with a value of $T_{a}=50^{\circ} \mathrm{C}$, the MITB $=T_{\text {con }}+\Delta \theta_{c}=$ $T_{a}+22=77{ }^{\circ} \mathrm{C}$, under no load. Under loaded conditions, in practice, $T_{c o n}>T_{a}$ (equation (7)), and hence MITB will be higher than that of an unloaded cable. This fact is shown in Figure 11 for the case of $T_{a}=25^{\circ} \mathrm{C}$ (for a higher sheath temperature, say $T_{a}{ }^{\prime}$, all that is required is to shift upward the curves of Figure 11 by an equal amount, $\left(T_{a}{ }^{\prime}-T_{a}\right)$ ).

Table 3. Comparison of MITB for different activation energies.

\begin{tabular}{|c|c|c|c|}
\hline S.No & $b(K)$ & MITB $(K)$ & $\begin{array}{l}\Delta \theta_{c} \\
\left.{ }^{\circ} C\right)\end{array}$ \\
\hline Cable 1 & 7600 & 320 & 22 \\
\hline Cable 2 & 10000 & 313 & 15 \\
\hline
\end{tabular}




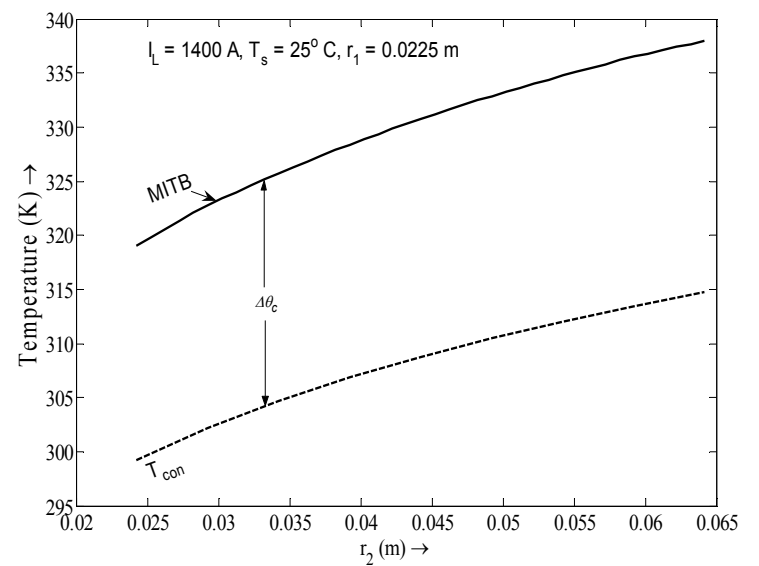

Figure 12. MITB and $T_{c o n}$ vs insulation thickness for constant load current.

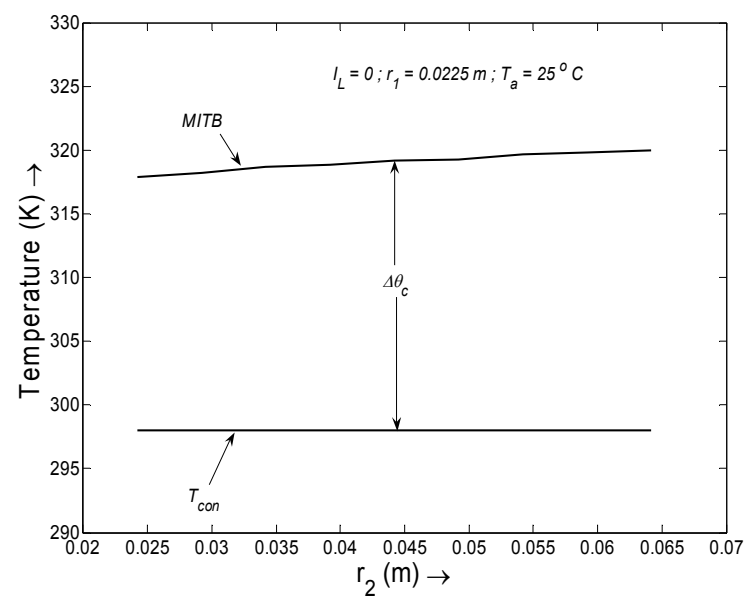

Figure 13. MITB and $T_{c o n}$ vs insulation thickness for no load.

\section{CONCLUSION}

In conclusion, the Authors' work presented in this paper, aims to appropriately specify limits on the maximum permissible temperature in cable insulation from the stand point of thermal failure. The following aspects are shown to be the more important considerations in dc cable design.

- The ITMV is shown to increase with the insulation thickness in spite of the fact that the average thermal breakdown strength and maximum insulation stress at breakdown decrease with it.
- A higher factor of safety for insulation thickness seems to provide deterrence for failure of cables against system over voltages.

- The temperature difference, $\Delta \theta_{c}$, may be used with advantage as an index for assessing a class of cable insulating materials besides a possibility of using it to predict the thermal breakdown.

\section{REFERENCES}

[1] C. K. Eoll, "Theory of Stress Distribution in Insulation of High Voltage DC Cables: Part I", IEEE Trans. Electr. Insul. Vol. 10, pp. 27-35, 1975.

[2] M. M. Fallou, "Perforration dielectrique par instabilite thermique des cables a courant continu", Revue Generale de LElectricite, pp. 693-695, 1959.

[3] S. Whitehead, Dielectric Breakdown of Solids, Oxford University Press, London, 1951

[4] J. J. O'Dwyer, The Theory of Dielectric Breakdown of Solids, Oxford University Press, London, 1964.

[5] Ch. C. Reddy and T. S. Ramu, "On the computation of Electric Field and Temperature Distribution in HV DC Cable insulation", IEEE Trans. Dielectr. Electr. Insul. Vol. 13, pp 1236-1244, 2006.

[6] J. M. Oudin and H. Thevenon, "DC cables determination of the gradient actually applied to the insulant and its experimental correlation with breakdown gradient", CIGRE, paper 208, 1966.

[7] F. H. Buller, "Calculation of Electrical stresses in the DC Cable insulation", IEEE Trans. Power App. Syst., pp. 1177-1178, 1967.

[8] R. A. Labozetta, "The Development of a High Voltage DC Cable", ERDA, EPRI, Final report, 1978.

[9] L. A. Dissado and J. C. Fothergill, Electrical degradation and breakdown in polymers, Peter Peregrinus, London, 1992.

[10] U. Riechert, R. Vogelsang, and J. Kindersberger, "Temperature Effect on DC Breakdown of Polyethylene Cables", 12th Intern. Sympos. High Voltage Engineering, ISH, Bangalore, India. pp. 537-540, 2001.

[11] M. N. Ozisik, Heat transfer - A Basic Approach, Mc.Graw Hill International Edition, 1985.

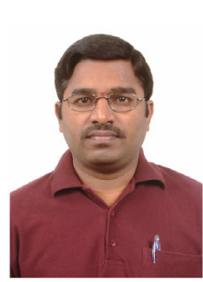

Ch. Chakradhar Reddy was born in Warangal, India. He received the B.E. degree (Electrical Engineering.) from the University College of Engineering, Osmania University, Hyderabad, India, and the M.E degree (Electrical Engineering), from Indian Institute of Science (IISc), Bangalore, India. Among others, his research interests include XLPE power cables, high voltage \& insulation engineering and control systems engineering. Currently, he is a research scholar (Ph.D.) in the Department of Electrical Engineering, IISc, India.

T. S. Ramu was born in Bangalore, India. He received the B.E. degree from Mysore University, Bangalore, India, the M.E. degree from IISc, Bangalore, and the Ph.D. degree from IIT Madras, India. Currently, he is a professor in the Department of High Voltage Engineering, IISc, India 